\title{
Depressive symptoms and C-reactive protein: The Cardiovascular Risk in Young Finns Study
}

\author{
MARKO ELOVAINIO ${ }^{1,2}$, LIISA KELTIKANGAS-J ÄRVINEN ${ }^{1}$, \\ LAURA PULKKI-R A BACK ${ }^{1}$, MIKA KIVIMÄKI ${ }^{1,3}$, SAMPSA PUTTONEN ${ }^{1}$, \\ LIISA VIIKARI ${ }^{5}$, LEENA RÄSÄNEN ${ }^{5}$, KRISTIINA MANSIKKANIEMI ${ }^{4}$, \\ JORMA VIIKARI ${ }^{5}$ AND OLLI T. RAITAKARI ${ }^{6 *}$
}

${ }^{1}$ Department of Psychology, University of Helsinki, Finland; ${ }^{2}$ National Research and Development Centre of Welfare and Health (STAKES), Helsinki, Finland; ${ }^{3}$ Finnish Institute of Occupational Health, Helsinki,

Finland ${ }^{4}$ Research Centre of Applied and Preventive Cardiovascular Medicine, University of Turku, Finland $;{ }^{5}$ Department of Medicine, University of Turku, Finland; ${ }^{6}$ Department of Clinical Physiology, University of Turku, Finland

\begin{abstract}
Background. We tested the hypothesis that depressive symptoms in healthy young adults would be associated with elevated levels of C-reactive proteins (CRP).

Method. We studied the association between depressive symptoms and CRP in 1201 young adults, as a part of the on-going population-based Cardiovascular Risk in Young Finns Study. Depressive symptoms were determined by responses to a revised version of Beck's Depression Inventory in 1992 and 2001. CRP and other known cardiac risk factors were measured in 2001.
\end{abstract}

Results. Higher depressive symptomatology in 1992 and in 2001 and their means score were related to higher CRP levels ( $B$ 's range from $0 \cdot 24$ to $0 \cdot 21, p<0 \cdot 001$ ). These relationships persisted after separate adjustments for various risk factors including sex, age, education, oral contraceptive use, dietary fat, physical activity, alcohol consumption, smoking status, LDL-cholesterol, HDLcholesterol, systolic blood pressure and history of acute infectious disease. Adjustments for obesity and triglycerides levels, however, somewhat attenuated the relationship between depressive symptoms and CRP.

Conclusions. We concluded that higher levels of depressive symptoms are associated with higher levels of CRP, but this association may largely be attributable to obesity or triglycerides.

\section{INTRODUCTION}

Depressive symptoms and even major depressive disorders are prevalent conditions in general populations. Lifetime prevalence of major depressive disorders in both USA and Western Europe was found to be around $13 \cdot 3-17 \cdot 1 \%$ (Kessler et al. 1994; Cassano \& Fava, 2002). Significant suffering, high morbidity and mortality, and psychosocial functional impairment are typically associated with depressive

\footnotetext{
* Address for correspondence: Olli T. Raitakari, M.D., Ph.D., Department of Clinical Physiology, University of Turku, Kiinamyllynkatu 4-8, Turku FIN-20521, Finland.

(Email: olli.raitakari@utu.fi)
}

disorders. Public health significance of depression has been stressed, due to the impact that depression has on the quality of life and especially on the course and mortality associated with other medical diseases.

It has been suggested that depression promotes (Maier \& Watkins, 1998) and maintains inflammatory responses by diminishing the immune system's sensitivity to the glucocorticoid hormones that are responsible for terminating the inflammatory response (Carney et al. 2002). The brain has two major outflow pathways by which it can control peripheral organs such as those of the immune system. Autonomic nervous system controls the activity of organs 
by releasing catecholamines (norepinephrine and epinephrine). The other pathway, the hypothalamus-pituitary-adrenal cortex (HPA) axis, involves feedback mechanisms between the hypothalamus and pituitary gland (Plaut, 1987). Depression has been shown to be associated with a dysregulation of the HPA axis characterized by increases in basal levels of adrenal glucocorticoids and a reduced potency of dexamethasone to suppress adrenal glucocorticoids (Gold et al. 1996). The pro-inflammatory cytokines, such as interleukin-1 (IL-1) and interleukin-6 (IL-6), are potent stimulators of the HPA axis (Tilders et al. 1993). Furthermore, depression has been argued to involve alterations in brain noradrenergic and serotonergic activity, effects, which may follow immune activation (Maier \& Watkins, 1998).

There is evidence that plasma levels of circulating IL-6 are elevated in depressed individuals (Sluzewska et al. 1995). Consistent with the idea that there is chronic immune activation during depression, depressed individuals show a blunted response to lipopolysaccharides (Carpenter et al. 2004). It has also been shown in animal studies, that production of IL-6 and other pro-inflammatory cytokines can be directly stimulated by negative emotions and stressful experiences (Kiecolt-Glaser et al. 2002a). IL-6 has a central role in promoting the production of C-reactive protein (CRP), a serological marker of systemic inflammation.

A growing body of evidence suggests that there is a relationship between depression and CRP. Depression has been shown to be associated with CRP in randomly sampled cardiovascular disease-free men $(n=453)$ and women $(n=400)$ living in the Attica area in Greece (Panagiotakos et al. 2004). Suarez (2004) showed that severity of depressive symptoms was significantly associated with elevations of CRP in 127 apparently healthy men and women. However, according to the results of Douglas et al. (2004) depressive symptoms were only weakly related to CRP in 696 army personnel, and this association was attenuated after the effect of body size was taken into account. Danner et al. (2003) found that a recent history of major depressive episodes was associated with elevated CRP levels in men aged 17-39 years. Similarly Ford \& Erlinger (2004) showed that compared to men without a history of depression, those who had recent depressive episodes had also higher CRP levels.

Depression may also contribute to cardiovascular heart disease by damaging the endothelium, by promoting maladaptive behaviour such as smoking (Miller et al. 1999), triggering dysregulation of the neurohormonal systems responsible for cortisol and catecholamine secretion (Plotsky et al. 1998) or increasing susceptibility to infection with latent pathogenesis that colonize the vessel wall (Herbert \& Cohen, $1993 a, b)$.

In this study, we examined the relationship between depressive symptoms and CRP in a population-based sample of young healthy adults. We took into account the effects of several potential confounding factors, such as age, sex, educational level, oral contraceptive use in women, body mass index (BMI), dietary fat, smoking, heavy alcohol use, physical activity, triglycerides, High-density lipoprotein (HDL)-cholesterol, Low-density lipoprotein (LDL)-cholesterol, systolic blood pressure and recent infectious disease. All of these have been shown to be associated with depressive symptoms or inflammation (Pearlin, 1989; Anda et al. 1990; Istvan et al. 1992; Kiecolt-Glaser et al. 2002 b; Miller et al. 2002).

\section{METHOD}

\section{Subjects and methods}

The participants were derived from the on-going population-based, Cardiovascular Risk in Young Finns Study. In this prospective, epidemiological study, a randomized sample of 3596 Finnish children and adolescents aged 3, 6, $9,12,15$, and 18 years at baseline in 1980 has been followed with repeated examinations from childhood to adulthood (Akerblom et al. 1991). Childhood and adolescent cardiovascular risk factors were measured in 1980. A total of 2370 responded to a survey questionnaire on psychosocial factors, such as depressive symptoms in 1992. Of the original sample, 2229 participants were re-examined in 2001 by measuring depressive symptoms, adulthood cardiovascular risk factors and CRP. The number of participants who completed the revised version of Beck's Depression Inventory (BDI; Beck \& Beck, 1972; Nurmi et al. 1997; Katainen et al. 1999) was 1496 in 1992 and 1741 in 2001. The 
sample with complete data (depression measured 1992 and in 2001) consisted of 488 men and 713 women who were 3-18 years of age at baseline in 1980. Those who had a diagnosis of diabetes mellitus, ischaemic heart disease or rheumatoid disease $(n=10)$ were excluded from the study sample. Compared to the eligible population at baseline, the participants in the present sample were more often women $\left(\chi^{2}=110 \cdot 24, \mathrm{df}=1, p<0 \cdot 001\right)$, but other baseline differences were small and statistically insignificant.

\section{CRP}

Serum high sensitive CRP (hsCRP) was analysed by an automated analyser (Olympus AU400; Olympus, USA) and a highly sensitive turbidimetric immunoassay kit ('CRP-UL' assay, Wako Chemicals, Neuss, Germany). Detection limit of the assay was $0.06 \mathrm{mg} / \mathrm{l}$. The inter-assay coefficient of variation was $3.33 \%$ at the mean level of $1.52 \mathrm{mg} / 1(n=116)$ and $2.65 \%$ at the mean level of $2.51 \mathrm{mg} / 1(n=168)$.

\section{Depressive symptoms}

Depressive symptoms were assessed by using a revised version of the BDI (Beck \& Beck, 1972; Katainen et al. 1999). The original version of the BDI is a 21-item questionnaire including four alternative statements for each item. In the present study, the subjects were asked to rate the 21 items (e.g. 'I often feel sad') on a 5-point scale (ranging from totally disagree $=1$, to totally agree $=5$ ). These items were the second mildest statements of the original items, and were selected because they were suggested to be the most effective ones to measure depression in the normal population (Nurmi et al. 1997; Katainen et al. 1999). The items tap negative mood, sadness, pessimism, decreased functioning such as indecisiveness and work inhibition, and somatic problems such as insomnia and fatigue. Cronbach's $\alpha$ reliability for the modified BDI was 0.90 in 1992, and 0.92 in 2001. The mean score of all items in both years were summed up to form a general depressive symptoms index, which was treated as a continuous variable.

\section{Potential confounding factors}

Potential confounding factors were measured in adolescence and adulthood a the same time as the other variables and included age, occupation, education, alcohol consumption, physical activity, diet (use of butter, margarine or vegetable oil as dietary fat), blood levels of LDL-cholesterol, HDL-cholesterol, triglycerides, total cholesterol, BMI, and systolic blood pressure, smoking, oral contraceptive pill use, and recent infectious disease (during the last 2 weeks). All measurements of lipid levels were performed in duplicate in the same laboratory. Standardized enzymatic methods were used for measuring levels of serum total cholesterol, triglycerides, and HDL-cholesterol. LDL-cholesterol concentration was calculated by the Friedewald formula (Friedewald et al. 1972). Blood pressure was measured with a standard mercury sphygmomanometer in 1980 and with random zero sphygmomanometer in 2001. The average of three blood pressure measurements was used in statistical analysis. Details of the methods have been reported elsewhere (Akerblom et al. 1991; Juonala et al. 2004; Raitakari et al. 2005).

\section{Statistical analysis}

The multivariate relationships between depressive symptoms and CRP were studied by linear regression analyses. Three types of depressive symptoms variables were used as the independent variable: (1) the depressive symptoms in 2001, (2) the mean depressive symptoms of 1992 and 2001 and (3) the depressive symptoms in 1992 adjusted for the change score (2001-1992) of depressive symptoms. The logtransformed CRP level in 2001 was the dependent variable. Statistical models were adjusted for, age, sex, education, physical activity, BMI, alcohol consumption, smoking status, LDLcholesterol, HDL-cholesterol, log-transformed triglycerides, systolic blood pressure and history of recent infectious disease. The regression models were fitted with logarithm of CRP and triglycerides because the distributions of these variables were skewed.

As the first set in the models, CRP measured in 2001 was regressed on age and depressive symptoms. Then in the following sets the regression between depressive symptoms and CRP was adjusted for age and sex, age, sex and physical activity, age, sex and dietary fat, age, sex and BMI, age, sex and alcohol consumption, age, sex and smoking status, age, sex 
Table 1. Characteristics of the study subjects $(n=1201)$

\begin{tabular}{|c|c|c|c|c|}
\hline Variable & $n$ & $\%$ & Mean (S.D.) & Range \\
\hline Age (yr) & & & $31 \cdot 5(5 \cdot 0)$ & $24 \cdot 0-39 \cdot 0$ \\
\hline \multicolumn{5}{|l|}{ Sex } \\
\hline Males & 488 & $40 \cdot 6$ & & \\
\hline Females & 713 & $59 \cdot 4$ & & \\
\hline \multicolumn{5}{|l|}{ Educational level } \\
\hline Elementary school & 164 & $13 \cdot 7$ & & \\
\hline High school & 789 & $65 \cdot 7$ & & \\
\hline College/university & 248 & $20 \cdot 6$ & & \\
\hline \multicolumn{5}{|l|}{ Body mass index } \\
\hline$<30 \mathrm{~kg} / \mathrm{m}^{2}$ & 1064 & $88 \cdot 6$ & & \\
\hline$\geqslant 30 \mathrm{~kg} / \mathrm{m}^{2}$ & 137 & $11 \cdot 4$ & & \\
\hline \multicolumn{5}{|l|}{ Dietary fat } \\
\hline Vegetable oil & 581 & $48 \cdot 4$ & & \\
\hline Margarine & 335 & $27 \cdot 9$ & & \\
\hline Butter & 285 & $23 \cdot 7$ & & \\
\hline \multicolumn{5}{|l|}{ Smoking } \\
\hline Non-smokers & 820 & $69 \cdot 4$ & & \\
\hline Smokers & 381 & $31 \cdot 6$ & & \\
\hline Physical activity ${ }^{\mathrm{a}}$ & & & $9 \cdot 7(2 \cdot 3)$ & $5 \cdot 0-16 \cdot 0$ \\
\hline Alcohol consumption ${ }^{\mathrm{b}}$ & & & $4 \cdot 5(1 \cdot 4)$ & $1-6$ \\
\hline Triglycerides $(\mathrm{mmol} / \mathrm{l})$ & & & $1 \cdot 26(0 \cdot 64)$ & $0 \cdot 40-3 \cdot 90$ \\
\hline HDL cholesterol (mmol/l) & & & $1 \cdot 31(0 \cdot 31)$ & $0 \cdot 31-2 \cdot 56$ \\
\hline LDL cholesterol $(\mathrm{mmol} / \mathrm{l})$ & & & $3 \cdot 24(0 \cdot 85)$ & $1 \cdot 00-7 \cdot 90$ \\
\hline Systolic blood pressure $(\mathrm{mmHg})$ & & & $116(13)$ & $80-166$ \\
\hline Recent infectious disease ${ }^{c}$ & 61 & $5 \cdot 1$ & & \\
\hline Depressive symptoms & & & $2 \cdot 1(0 \cdot 7)$ & $1 \cdot 0-4 \cdot 5$ \\
\hline C-reactive protein $(\mathrm{mg} / \mathrm{l})$ & & & $1 \cdot 95(4 \cdot 26)$ & $0 \cdot 05-64 \cdot 9$ \\
\hline
\end{tabular}

HDL, High-density lipoprotein; LDL, low-density lipoprotein.

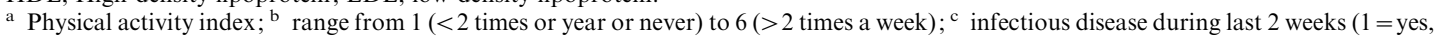
$0=$ no) $;{ }^{\mathrm{d}}$ agreement on scale $1-5$ of having depressive symptoms.

and LDL-cholesterol, age, sex and HDLcholesterol, age, sex and triglycerides, age, sex and systolic blood pressure, age, sex and recent infectious disease and in women oral contraceptive use. Finally the regression between depressive symptoms and CRP was adjusted for all of the aforementioned variables.

This strategy was applied to examine (i) whether level of depressive symptoms (measured at the same time or 9 years before) were associated with CRP, (ii) whether depressive symptoms were associated with CRP after adjustment for other known risk factors and (iii) whether some other risk factors were responsible for the relationship between depressive symptoms and CRP.

The statistical tests were performed with SAS version 9.01 (SAS Institute, Cary, NC, USA), and statistical significance was inferred at a two-tailed $p$ value of $<0.05$. We did not define any arbitrary cut-points to exclude subjects with high hsCRP values (for example values
$>10 \mathrm{mg} / \mathrm{l})$. We additionally used a dichotomous definition of CRP $(<3 v . \geqslant 3)$ and calculated the odds ratios (ORs) for having elevated CRP levels.

\section{RESULTS}

The characteristics of study subjects are shown in Table 1. Slightly more than half of the respondents were women, one fifth had college/ university education, more than $10 \%$ were obese, and almost half used vegetable oil as their dietary fat. Of the women in the sample $26 \%$ used oral contraceptives. Univariate relationships (Person correlations) between other potential risk factors, depressive symptoms and CRP are shown in Table 2. A significant relationship was found between CRP and sex $\quad(r=-0 \cdot 12, \quad p<0 \cdot 001), \quad$ BMI $\quad(r=0 \cdot 20$, $p<0.001)$, triglycerides $(r=0.14, \quad p<0 \cdot 001)$, HLD-cholesterol $(r=0.07, p<0 \cdot 01)$, and recent infectious disease $(r=0 \cdot 16, p<0 \cdot 001)$. Among 
Table 2. Zero-order correlations among study variables $(n=1201)$

\begin{tabular}{|c|c|c|c|c|c|c|c|c|c|c|c|c|c|c|c|c|}
\hline Variable & 1 & 2 & 3 & 4 & 5 & 6 & 7 & 8 & 9 & 10 & 11 & 12 & 13 & 14 & 15 & 16 \\
\hline 1. Age (yr) & $1 \cdot 00$ & & & & & & & & & & & & & & & \\
\hline $\begin{array}{l}\text { 2. Sex }(1=\text { woman, } \\
2=\operatorname{man})\end{array}$ & $0 \cdot 00$ & $1 \cdot 00$ & & & & & & & & & & & & & & \\
\hline 3. Educational level & $0 \cdot 04$ & $0 \cdot 03$ & $1 \cdot 00$ & & & & & & & & & & & & & \\
\hline 4. Body mass index & $0 \cdot 05$ & $0 \cdot 00$ & -0.07 & $1 \cdot 00$ & & & & & & & & & & & & \\
\hline 5. Dietary fat & $0 \cdot 08$ & $0 \cdot 12$ & $-0 \cdot 13$ & 0.06 & 1.00 & & & & & & & & & & & \\
\hline 6. Smoking & -0.06 & $0 \cdot 14$ & $-0 \cdot 12$ & $0 \cdot 04$ & $0 \cdot 08$ & $1 \cdot 00$ & & & & & & & & & & \\
\hline 7. Alcohol consumption & 0.06 & $-0 \cdot 37$ & 0.07 & $0 \cdot 01$ & $0 \cdot 02$ & $-0 \cdot 37$ & $1 \cdot 00$ & & & & & & & & & \\
\hline 8. Physical activity & $-0 \cdot 14$ & 0.03 & $0 \cdot 03$ & $-0 \cdot 11$ & $-0 \cdot 08$ & -0.09 & $-0 \cdot 10$ & $1 \cdot 00$ & & & & & & & & \\
\hline 9. Triglycerides ${ }^{\mathrm{a}}$ & 0.00 & $0 \cdot 19$ & 0.03 & $0 \cdot 26$ & 0.02 & $0 \cdot 08$ & $-0 \cdot 13$ & -0.04 & $1 \cdot 00$ & & & & & & & \\
\hline 10. HDL cholesterol & $0 \cdot 02$ & $-0 \cdot 35$ & -0.02 & $-0 \cdot 24$ & -0.06 & $-0 \cdot 13$ & 0.03 & 0.07 & $-0 \cdot 28$ & $1 \cdot 00$ & & & & & & \\
\hline 11. LDL cholesterol & $0 \cdot 19$ & $0 \cdot 16$ & 0.01 & $0 \cdot 16$ & $0 \cdot 10$ & 0.03 & $-0 \cdot 01$ & $-0 \cdot 13$ & $0 \cdot 26$ & -0.03 & $1 \cdot 00$ & & & & & \\
\hline 12. Systolic blood pressure & $0 \cdot 08$ & $0 \cdot 33$ & -0.01 & $0 \cdot 23$ & 0.07 & $-0 \cdot 00$ & $-0 \cdot 15$ & 0.06 & $0 \cdot 26$ & $-0 \cdot 13$ & $0 \cdot 12$ & $1 \cdot 00$ & & & & \\
\hline $\begin{array}{l}\text { 13. Recent infectious } \\
\text { disease }\end{array}$ & -0.03 & $-0 \cdot 05$ & $-0 \cdot 04$ & $0 \cdot 04$ & $0 \cdot 02$ & 0.03 & -0.02 & $0 \cdot 01$ & $0 \cdot 06$ & $-0 \cdot 05$ & $0 \cdot 02$ & $0 \cdot 02$ & $1 \cdot 00$ & & & \\
\hline 14. C-reactive protein & $0 \cdot 00$ & $-0 \cdot 12$ & -0.03 & $0 \cdot 20$ & $0 \cdot 03$ & $-0 \cdot 01$ & $-0 \cdot 00$ & -0.04 & $0 \cdot 14$ & $0 \cdot 07$ & $-0 \cdot 02$ & $0 \cdot 03$ & $0 \cdot 16$ & 1.00 & & \\
\hline $\begin{array}{l}\text { 15. Depressive symptoms } \\
1992\end{array}$ & -0.07 & $-0 \cdot 15$ & $-0 \cdot 10$ & 0.09 & -0.03 & $0 \cdot 00$ & 0.01 & $-0 \cdot 11$ & $0 \cdot 03$ & -0.02 & -0.04 & -0.02 & 0.03 & $0 \cdot 08$ & $1 \cdot 00$ & \\
\hline $\begin{array}{l}\text { 16. Depressive symptoms } \\
2001\end{array}$ & $0 \cdot 03$ & $-0 \cdot 14$ & -0.01 & $0 \cdot 12$ & $-0 \cdot 04$ & $0 \cdot 00$ & 0.02 & $-0 \cdot 18$ & 0.09 & $0 \cdot 01$ & $0 \cdot 01$ & $0 \cdot 01$ & $-0 \cdot 01$ & $0 \cdot 09$ & 0.53 & $1 \cdot 00$ \\
\hline
\end{tabular}

HDL, High-density lipoprotein; LDL, low-density lipoprotein.

${ }^{a}$ Logarithmically transformed, all correlations $>0.06$ are statistically significant and $>0.09$ are statistically highly significant.

women there was also a significant relationship between oral contraceptive use and CRP $(B=0 \cdot 91, t=9 \cdot 77, p<0 \cdot 001)$. Depressive symptoms in 1992 and in 2001 were significantly related to sex $(r=-0 \cdot 15$ and $-0 \cdot 14, p<0 \cdot 001)$, BMI $(r=0.09$ and $r=0 \cdot 12, p<0 \cdot 001)$, physical activity $(r=-0.11$ and $r=-0.18, p<0.001)$ and CRP $(r=0.08, \quad p<0.01$ and $r=0.09$, $p<0.001)$. Depressive symptoms in 1992 was additionally related to age $(r=-0 \cdot 07, p<0 \cdot 01)$ and depressive symptoms in 2001 to triglycerides $(r=0.09, p<0.001)$.

The results of the hierarchical regression analysis with CRP as the dependent variable and depressive symptoms as the independent variable are shown in Table 2 . Because sex had no statistically significant interaction effect in the relationship between CRP and depressive symptoms $(B=-0.06, t=-0.44, p=0.662)$ the regression analyses were made simultaneously for both sexes.

Higher level of depressive symptoms in 2001 was related to higher CRP $(B=0 \cdot 21, t=3 \cdot 89$, $p<0.001)$ and this association persisted with separate adjustments for many of the single risk factors (demographic characteristics, cardiovascular risk factors, infectious disease). We additionally adjusted the relationship between depressive symptoms and CRP for oral contraceptive use among women, but the association between depressive symptoms and CRP remained significant $(B=0 \cdot 14, t=2 \cdot 38$, $p=0.017$ ) After adjustment for age, sex and obesity the relationship between depressive symptoms and high CRP was no longer statistically significant $(B=0 \cdot 10, t=1 \cdot 88, p=0 \cdot 060)$. Also adjustment for log-transformed triglycerides attenuated the relationship $(B=0.09$, $t=1.77, \quad p=0.077$ ). After adjustment for all other risk factors the association between depressive symptoms in 2001 and CRP was not statistically significant $(B=0.05, \quad t=0.92$, $p=0.359)$. The full model explained $21 \%$ of the total variance of CRP (Table 3 ).

Similarly, the mean level of depressive symptoms in 1992 and $2001(B=0 \cdot 25, t=3 \cdot 90$, $p<0.001)$ and the level of depressive symptoms in 1992 adjusted for the change of depressive symptoms between 1992 and $2001(B=0 \cdot 24$, $t=3 \cdot 73, p<0 \cdot 001)$ were significantly related to CRP. These relationships were attenuated after adjustment for BMI or triglycerides or all other risk factors simultaneously. Both of the full models (including all risk factors) explained $22 \%$ of the total variance of CRP (Table 3 ).

There was also a relationship between dichotomous CRP $(<3 v . \geqslant 3)$ and linear trend of depressive symptoms (cut-off points for high 
Table 3. Unstandardized regression coefficients $(B)$ of depressive symptoms on $C R P$ in men and women $(n=1201)$. Adjusted for age, sex, education, body mass index, physical activity, alcohol consumption, smoking status, LDL-cholesterol, HDL-cholesterol, triglycerides, systolic blood pressure and recent infectious disease

\begin{tabular}{|c|c|c|c|c|c|c|c|c|c|}
\hline & \multicolumn{3}{|c|}{ Model 1} & \multicolumn{3}{|c|}{ Model 2} & \multicolumn{3}{|c|}{ Model 3} \\
\hline & $B$ & $t$ value & $p$ value & $B$ & $t$ value & $p$ value & $B$ & $t$ value & $p$ value \\
\hline Unadjusted & $0 \cdot 21$ & $3 \cdot 74$ & $<0 \cdot 001$ & $0 \cdot 25$ & $3 \cdot 90$ & $<0.001$ & $0 \cdot 24$ & $3 \cdot 73$ & $<0.001$ \\
\hline \multicolumn{10}{|l|}{ Adjusted for } \\
\hline 1. Age & $0 \cdot 21$ & $3 \cdot 77$ & $<0 \cdot 001$ & $0 \cdot 25$ & $3 \cdot 89$ & $<0 \cdot 001$ & $0 \cdot 24$ & $3 \cdot 69$ & $<0 \cdot 001$ \\
\hline 2. Age and sex, & $0 \cdot 16$ & $3 \cdot 06$ & $0 \cdot 002$ & $0 \cdot 19$ & $2 \cdot 84$ & $0 \cdot 005$ & $0 \cdot 17$ & $2 \cdot 64$ & $0 \cdot 008$ \\
\hline 4. Age, sex, and body mass index & $0 \cdot 10$ & $1 \cdot 88$ & $0 \cdot 060$ & $0 \cdot 10$ & $1 \cdot 60$ & $0 \cdot 109$ & $0 \cdot 09$ & $1 \cdot 44$ & $0 \cdot 149$ \\
\hline 5. Age, sex, and dietary fat & $0 \cdot 16$ & $3 \cdot 06$ & $0 \cdot 002$ & $0 \cdot 19$ & $2 \cdot 84$ & $0 \cdot 005$ & $0 \cdot 17$ & $2 \cdot 64$ & $0 \cdot 008$ \\
\hline 6. Age, sex, and smoking & $0 \cdot 16$ & $3 \cdot 03$ & $0 \cdot 003$ & $0 \cdot 19$ & $2 \cdot 84$ & $0 \cdot 005$ & $0 \cdot 18$ & $2 \cdot 65$ & $0 \cdot 008$ \\
\hline 7. Age, sex, and heavy alcohol use & $0 \cdot 16$ & $2 \cdot 98$ & $0 \cdot 003$ & $0 \cdot 18$ & $2 \cdot 74$ & $0 \cdot 006$ & $0 \cdot 17$ & $2 \cdot 54$ & $0 \cdot 011$ \\
\hline 8. Age, sex, and physical activity & $0 \cdot 15$ & $2 \cdot 73$ & $0 \cdot 006$ & $0 \cdot 17$ & $2 \cdot 53$ & $0 \cdot 012$ & $0 \cdot 16$ & $2 \cdot 36$ & $0 \cdot 018$ \\
\hline 9. Age, sex, and triglycerides & $0 \cdot 09$ & $1 \cdot 77$ & $0 \cdot 077$ & $0 \cdot 11$ & $1 \cdot 71$ & $0 \cdot 009$ & $0 \cdot 10$ & $1 \cdot 62$ & $0 \cdot 105$ \\
\hline 10. Age, sex, and HDL-cholesterol & $0 \cdot 16$ & $2 \cdot 95$ & $0 \cdot 003$ & $0 \cdot 18$ & $2 \cdot 69$ & $0 \cdot 007$ & $0 \cdot 16$ & $2 \cdot 48$ & $0 \cdot 013$ \\
\hline 11. Age, sex, and LDL-cholesterol & $0 \cdot 16$ & $2 \cdot 99$ & $0 \cdot 003$ & $0 \cdot 18$ & $2 \cdot 81$ & $0 \cdot 005$ & $0 \cdot 17$ & $2 \cdot 63$ & $0 \cdot 009$ \\
\hline 13. Age, sex, and recent infectious disease & $0 \cdot 17$ & $3 \cdot 15$ & $0 \cdot 002$ & $0 \cdot 19$ & $2 \cdot 90$ & $0 \cdot 004$ & $0 \cdot 18$ & $2 \cdot 69$ & $0 \cdot 007$ \\
\hline $\begin{array}{l}\text { 14. Age, sex, educational level, body } \\
\text { mass index, dietary fat, smoking, } \\
\text { heavy alcohol use, physical activity, } \\
\text { triglycerides, HDL-cholesterol, } \\
\text { LDL-cholesterol systolic blood } \\
\text { pressure and recent infectious disease }\end{array}$ & $0 \cdot 05$ & 0.92 & $0 \cdot 359$ & $0 \cdot 05$ & $0 \cdot 78$ & $0 \cdot 437$ & $0 \cdot 04$ & $0 \cdot 70$ & $0 \cdot 481$ \\
\hline
\end{tabular}

CRP, C-reactive protein; LDL, low-density lipoprotein; HDL, high-density lipoprotein.

Regression coefficient values indicate one unit change in log CRP (mg/l) for one unit of change in depressions score (agreement of having depressive symptoms on a scale of 1 (totally disagree) to 5 (totally agree).

Model 1: Depressive symptoms measured in 2001 as an independent variable. Model 2: Depressive symptoms measured as a mean of depressive symptoms in 1992 and 2001 as an independent variable. Model 3: Depressive symptoms measured in 1992 and adjusted for depressive symptoms change between 1992 and 2001 as an independent variable.

and low refers to - S.D. and + S.D.) in 2001 [OR $1 \cdot 07,95 \%$ confidence interval $(\mathrm{CI}) 1 \cdot 02-1 \cdot 11]$ of depressive mean score of 1992 and 2001 (OR $1 \cdot 06,95 \%$ CI $1 \cdot 02-1 \cdot 10)$ and of depressive symptoms in 1992 adjusted for change score (OR 1·06, 95\% CI 1·02-1·11) (Table 4).

We also dichotomized the mean level of depressive symptoms in 1992 and 2001 into two categories low and high (median split). In the low depressive symptoms group the average CRP level was 1.67 (S.D. $=3.78$ ) and in the high depressive symptoms group $2 \cdot 22$ (S.D. $=4 \cdot 47$ ). The difference was statistically significant $(t=-2 \cdot 31, \mathrm{df}=1209, p=0 \cdot 023)$.

\section{DISCUSSION}

We found that higher level of depressive symptoms, measured as depressive symptoms in 2001, as an average score of depressive symptoms in 1992 and 2001, or as depressive symptoms in 1992, adjusted for depressive symptoms change during 1992 and 2001 were all related to higher levels of CRP in healthy young adults. These relationships persisted after taking into account the effects of a variety of risk factors. However, obesity and triglycerides seemed to attenuate the relationships between depressive symptoms and CRP. Of the other risk factors included in the final model, female sex, low educational level, physical inactivity, obesity, triglycerides, recent infectious disease, and in women the use of oral contraceptives, had significant relationships with CRP.

The present findings are in line with the previous studies suggesting an association between depressive symptoms and CRP (Danner et al. 2003; Douglas et al. 2004; Ford \& Erlinger, 2004; Panagiotakos et al. 2004; Suarez, 2004). In some of the studies obesity has been shown to be mainly responsible for this association (Miller et al. 2002; Douglas et al. 2004). Our results offered partial support for the idea that triglycerides, in addition to obesity, are 
Table 4. Odds ratios (95\% confidence intervals) of CRP by levels of depressive symptoms adjusted for age and sex among participants with no diagnosed depression at baseline. Cut-off points for low and high levels of depressive symptoms refers to - S.D. and + S.D. respectively

\begin{tabular}{cccc}
\hline \hline & Model 1 & Model 2 & Model 3 \\
\hline Depressive symptoms (low $v$. high) & $1 \cdot 07(1 \cdot 02-1 \cdot 11)$ & $1 \cdot 06(1 \cdot 02-1 \cdot 10)$ & $1 \cdot 06(1 \cdot 02-1 \cdot 11)$ \\
\hline \hline
\end{tabular}

Model 1: Depressive symptoms measured in 2001. Model 2: Depressive symptoms measured as a mean of depressive symptoms in 1992 and 2001. Model 3: Depressive symptoms measured in 1992 and adjusted for depressive symptoms change between 1992 and 2001.

potential confounders in the relationship between depressive symptoms and CRP. However, it has recently been suggested that there may not be an independent association between CRP and the plasma lipid-lipoprotein profile but that this association is largely mediated by the increased visceral adipose tissue accumulation (Piche et al. 2005). It has also been suggested that that there is a synergistic effect of depressive mood and obesity on chronic low level inflammation (Ladwig et al. 2003).

Despite the fact that depression is associated with interference with specific immunity and increased disease susceptibility (Herbert $\&$ Cohen, 1993b), there are findings that support the argument that non-specific immune activation and cytokines are involved in the aetiology or symptomology of depression. Infectious diseases involve immune activation and cytokine release, and depression has been consistently reported with psychological disturbance in such diseases (Meijer et al. 1988). Finally, in experiments where cytokines have been administered to humans, depressed mood has frequently been observed as a consequence (Fent \& Zbinden, 1987).

Depression and obesity have been shown to associate even among young adults (Herva et al. 2006). A complementary explanation on the interrelations of CRP, depression and obesity comes from the stress research. Behavioural and endocrine changes produced by stressors are very similar to those produced by immune activation. Coe et al. (1988) suggested that stressors might activate macrophages and the effect was observed long after the stressor was terminated. Stress stimulates the brain to produce messengers, such as IL-1, that act on macrophages. IL-1 administered into the brain produces large increases in plasma IL-6 (Desimoni et al. 1990) a potential key mediator of the peripheral sickness changes observed after exposure to stressors. The end-hormones of the HPA axis, glucocorticoids, have direct as well as insulinmediated effects on adipose tissue, ultimately promoting visceral adiposity and while directly secreted by peripheral nerves glucocorticoids stimulate local inflammation (Maier \& Watkins, 1998).

A previous twin-study shows that the link between CRP and adiposity is robust to other risk factors, including genetic influences (Greenfield et al. 2004). CRP is mainly produced by the liver and to some extent by the adipose tissue and released in response to cytokine production (Heinrich et al. 1990). The second explanation, in addition to insulin resistance, may be increased IL-6 production by adipocytes, stimulating hepatic CRP production (Yudkin et al. 1999).

Depression may be associated with the activation of innate immunity in a similar manner to stress. The factor that causes depression (stressful life-events, negative cognitions about the self) may have access to the same neural circuitry that has evolved to mediate sickness. Some of the symptoms of depression may, therefore, be the result of this process and represent essentially sickness responses. The circuit is bi-directional, and so peripheral immune cells should also become activated, and the products of these activated immune cells in turn provide signals to the brain. It is conceivable that this positive feedback circuit could help to maintain depression (Maier \& Watkins, 1998).

CRP may represent a key factor in explaining the relationship between depressive symptoms and cardiovascular heart disease. Coronary artery disease especially is increasingly being viewed as a chronic inflammatory response to 
injuries of the vascular endothelium (Ross, 1999). Depression may affect health behaviour, neurohormonal systems or increase susceptibility to infection (Carney et al. 2002). Each of these processes are potentially damaging the endothelium, thereby triggering inflammatory processes that may lead to the progression of atherosclerosis.

In interpreting the present results, it is important to note some limitations. Only $34 \%$ of the baseline cohort persisted to follow-up. However, generalizability should not be substantially affected because the participants were largely similar to those who were lost. Women were over-represented but this is an unlikely source of bias because models were adjusted for sex. Although the Cardiovascular Risk in Young Finns Study is a population-based, longitudinal study with a large sample of six age cohorts, the present analysis was restricted to men and women aged 24-39 years at the time of the CRP measurement. Thus, the association between depressive symptoms and CRP needs to be studied using population- based samples with larger age variety.

The association between the depressive symptoms and higher CRP remained after adjusting for a number of other risk factors measured in adolescence and in adulthood. There are of course other potential cofounders that were not measured in this study. Future studies need to investigate their effects in the relationship between depression and CRP. The measure of depressive symptoms used in this study was a revised version of the widely used BDI. The subjects were asked to rate the second mildest statements of the original version on a 5-point scale, and thus we were not able to define any clinically significant cut point for depression. However, this scale was selected because it has been suggested to be an effective one to measure depressive symptoms in the nonclinical population (Nurmi et al. 1997; Katainen et al. 1999).

In summary, the present study demonstrates that depressive mood may contribute to the inflammation process and obesity or obesityrelated processes may be partly responsible for this association. Our study thus contributes to the growing body of evidence that provides support for the hypothesis that depression may exert adverse effects on physical health.

\section{ACKNOWLEDGEMENTS}

This study was financially supported by the Academy of Finland (grant nos. 53392 and 34316), the Social Insurance Institution of Finland, the Turku University Foundation, the Juho Vainio Foundation, the Yrjö Jahnsson Foundation, Special Federal grants for the Turku University Hospital, the Finnish Foundation of Cardiovascular Research, the Lydia Maria Julin Foundation and the Finnish Cultural Foundation.

\section{DECLARATION OF INTEREST}

None.

\section{REFERENCES}

Akerblom, H. K., Uhari, M., Pesonen, E., Dahl, M., Kaprio, E. A. Nuutinen, E. M., Pietikäinen, M., Salo, M. K., Aromaa, A., Kannas, L., Keltikangas-Järvinen, L., Kuusela, V., Räsänen, L., Rönnemaa, T., Knip, M., Telama, R., Välimäki, I., Pyörälä, K. \& Viikari, J. (1991). Cardiovascular risk in young Finns. Annals of Medicine 23, 35-39.

Anda, R. F., Williamson, D. F., Escobedo, L. G., Mast, E. E., Giovino, G. A. \& Remington, P. L. (1990). Depression and the dynamics of smoking. A national perspective. Journal of the American Medical Association 264, 1541-1545.

Beck, A. T. \& Beck, R. W. (1972). Screening depressed patients in family practice. A rapid technic. Postgraduate Medicine 52, 81-85.

Carney, R. M., Freedland, K. E., Miller, G. E. \& Jaffe, A. S. (2002). Depression as a risk factor for cardiac mortality and morbidity: a review of potential mechanisms. Journal of Psychosomatic Research 53, 897-902.

Carpenter, L. L., Heninger, G. R., Malison, R. T., Tyrka, A. R. \& Price, L. H. (2004). Cerebrospinal fluid interleukin (IL)-6 in unipolar major depression. Journal of Affective Disorders 79, 285-289.

Cassano, P. \& Fava, M. (2002). Depression and public health: an overview. Journal of Psychosomatic Research 53, 849-857.

Coe, C. L., Rosenberg, L. T. \& Levine, S. (1988). Prolonged effect of psychological disturbance on macrophage chemiluminescence in the squirrel monkey. Brain Behavior Immunity 2, 151-160.

Danner, M., Kasl, S. V., Abramson, J. L. \& Vaccarino, V. (2003) Association between depression and elevated C-reactive protein. Psychosomatic Medicine 65, 347-356.

Desimoni, J., Bibiloni, A. G., Massolo, C. P. \& Renteria, M. (1990). Hyperfine interactions in the cubic semiconductor CdO. Physical Review 41, 1443-1446.

Douglas, K. M., Taylor, A. J. \& O'Malley, P. G. (2004). Relationship between depression and C-reactive protein in a screening population. Psychosomatic Medicine 66, 679-683.

Fent, K. \& Zbinden, G. (1987). Toxicity of interferon and interleukin. Trends in Pharmacological Sciences 8, 100-105.

Ford, D. E. \& Erlinger, T. P. (2004). Depression and C-reactive protein in US adults: data from the Third National Health and Nutrition Examination Survey. Archives of Internal Medicine 164, $1010-1014$.

Friedewald, W. T., Levy, R. I. \& Fredrickson, D. S. (1972). Estimation of the concentration of low-density lipoprotein cholesterol in plasma, without use of the preparative ultracentrifuge. Clinical Chemistry 18, 499-502. 
Gold, P. W., Wong, M. L., Chrousos, G. P. \& Licinio, J. (1996). Stress system abnormalities in melancholic and atypical depression: molecular, pathophysiological, and therapeutic implications. Molecular Psychiatry 1, 257-264

Greenfield, J. R., Samaras, K., Jenkins, A. B., Kelly, P. J., Spector, T. D., Gallimore, J. R., Pepys, M. B. \& Campbell, L. V. (2004). Obesity is an important determinant of baseline serum C-reactive protein concentration in monozygotic twins, independent of genetic influences. Circulation 109, 3022-3028.

Heinrich, P. C., Castell, J. V. \& Andus, T. (1990). Interleukin-6 and the acute phase response. Biochemical Journal 265, 621-636.

Herbert, T. B. \& Cohen, S. (1993a). Depression and immunity: a meta-analytic review. Psychological Bulletin 113, 472-486.

Herbert, T. B. \& Cohen, S. (1993 b). Stress and immunity in humans: a meta-analytic review. Psychosomatic Medicine 55, 364-379.

Herva, A., Laitinen, J., Miettunen, J., Veijol, J., Karvonen, J. T., Läksy, K. \& Joukamaa, M. (2006). Obesity and depression: results from longitudinal Northern Finland 1966 Birth Cohohrt Study. International Journal of Obesity 30, 520-527.

Istvan, J., Zavela, K. \& Weidner, G. (1992). Body weight and psychological distress in NHANES I. International Journal of Obesity Related Metabolic Disorders 16, 999-1003.

Juonala, M., Viikari, J. S., Hutri-Kahonen, N., Pietikainen, M., Jokinen, E., Taittonen, L., Marniemi, J., Ronnemaa, T. \& Raitakari, O. T. (2004). The 21-year follow-up of the Cardiovascular Risk in Young Finns Study: risk factor levels, secular trends and east-west difference. Journal of Internal Medicine $\mathbf{2 5 5}$, $457-468$.

Katainen, S., Räikkönen, K. \& Keltikangas-Järvinen, L. (1999). Adolescent temperament, perceived social support, and depressive tendencies as predictors of depressive tendencies in young adulthood. European Journal of Personality 13, 183-207.

Kessler, R. C., McGonagle, K. A., Zhao, S., Nelson, C. B., Hughes, M., Eshleman, S., Wittchen, H. U. \& Kendler, K. S. (1994). Lifetime and 12-month prevalence of DSM-III-R psychiatric disorders in the United States. Results from the National Comorbidity Survey. Archives of General Psychiatry 51, 8-19.

Kiecolt-Glaser, J. K., McGuire, L., Robles, T. F. \& Glaser, R. (2002a). Emotions, morbidity, and mortality: new perspectives from psychoneuroimmunology. Annual Review of Psychology 53, $83-107$

Kiecolt-Glaser, J. K., McGuire, L., Robles, T. F. \& Glaser, R. $(2002 b)$. Psychoneuroimmunology and psychosomatic medicine: back to the future. Psychosomatic Medicine 64, 15-28.

Ladwig, K. H., Marten-Mittag, B., Lowel, H., Doring, A. \& Koenig, W. (2003). Influence of depressive mood on the association of CRP and obesity in 3205 middle aged healthy men. Brain, Behavior \& Immunity 17, 268-275.

Maier, S. F. \& Watkins, L. R. (1998). Cytokines for psychologists: implications of bidirectional immune-to-brain communication for understanding behavior, mood, and cognition. Psychological Review 105, 83-107.
Meijer, A., Zakay-Rones, Z. \& Morag, A. (1988). Post-influenzal psychiatric disorder in adolescents. Acta Psychiatrica Scandinavica 78, 176-181.

Miller, G. E., Cohen, S. \& Herbert, T. B. (1999). Pathways linking major depression and immunity in ambulatory female patients. Psychosomatic Medicine 61, 850-860.

Miller, G. E., Cohen, S. \& Ritchey, A. K. (2002). Chronic psychological stress and the regulation of pro-inflammatory cytokines: a glucocorticoid-resistance model. Health Psychology 21, 531-541.

Nurmi, J. E., Toivonen, S., Salmela-Aro, K. \& Eronen, S. (1997) Social strategies and loneliness. Journal of Social Psychology 137, 764-777.

Panagiotakos, D. B., Pitsavos, C., Chrysohoou, C., Tsetsekou, E., Papageorgiou, C., Christodoulou, G. \& Stefanadis, C. (2004). Inflammation, coagulation, and depressive symptomatology in cardiovascular disease-free people; the ATTICA study. European Heart Journal 25, 492-499.

Pearlin, L. I. (1989). The sociological study of stress. Journal of Health and Social Behavior 30, 241-256.

Piche, M. E., Lemieux, S., Weisnagel, S. J., Corneau, L., Nadeau, A. \& Bergeron, J. (2005). Relation of high-sensitivity C-reactive protein, interleukin-6, tumor necrosis factor-alpha, and fibrinogen to abdominal adipose tissue, blood pressure, and cholesterol and triglyceride levels in healthy postmenopausal women. American Journal of Cardiology 96, 92-97.

Plaut, M. (1987). Lymphocyte hormone receptors. Annual Review of Immunology 5, 621-669.

Plotsky, P. M., Owens, M. J. \& Nemeroff, C. B. (1998). Psychoneuroendocrinology of depression. Hypothalamic-pituitaryadrenal axis. Psychiatry Clinical North America 21, 293-307.

Raitakari, M., Mansikkaniemi, K., Marniemi, J., Viikari, J. \& Raitakari, O. T. (2005). Distribution and determinants of serum high-sensitive C-reactive protein in population of young adults. The cardiovascular Risk in Young Finns Study. Journal of Internal Medicine 258, 428-434.

Ross, R. (1999). Atherosclerosis is an inflammatory disease. American Heart Journal 138, S419-S420.

Sluzewska, A., Rybakowski, J. K., Laciak, M., Mackiewicz, A. Sobieska, M. \& Wiktorowicz, K. (1995). Interleukin-6 serum levels in depressed patients before and after treatment with fluoxetine. Annual New York Academy of Science 762, 474-476.

Suarez, E. C. (2004). C-reactive protein is associated with psychological risk factors of cardiovascular disease in apparently healthy adults. Psychosomatic Medicine 66, 684-691.

Tilders, F. J., Schmidt, E. D. \& de Goeij, D. C. (1993). Phenotypic plasticity of CRF neurons during stress. Annual New York Academy of Science 697, 39-52.

Yudkin, J. S., Stehouwer, C. D., Emeis, J. J. \& Coppack, S. W. (1999). C-reactive protein in healthy subjects: associations with obesity, insulin resistance, and endothelial dysfunction: a potential role for cytokines originating from adipose tissue? Arteriosclerosis, Thrombosis \& Vascular Biology 19, 972-978. 